\title{
The Yoga Boom in Western Society: Practitioners’ Spiritual vs. Physical Intentions and Their Impact on Psychological Wellbeing
}

Itai Ivtzan* and Sivaja Jegatheeswaran

Department of Psychology, University of East London, UK

\begin{abstract}
Whilst some scholars endorse the idea that yoga in the Western world is predominantly a postural practice, others argue that this Western manifestation, like the historically Indian tradition of yoga, could cultivate transcendental states of spirituality. This study's methodology compared practitioners' spiritual and physical intentions and their transformation over time. Furthermore, it was predicted that practitioners' psychological wellbeing (satisfaction with life and meaning in life) would vary as a function of their intention type. 235 yoga practitioners in the UK responded to measurements of their intentions and wellbeing. The results indicated that practitioners had greater initial and continued physical intentions than spiritual intentions. However, spiritual intentions become more salient over time, suggesting that Western yoga can cultivate spirituality. Results also showed that practitioners with spiritual intentions reported significantly higher psychological wellbeing. This has important implications for future conceptualizations of the practice, suggesting that Western societies should place a greater emphasis on the Indian-rooted spiritual aspect of yoga.
\end{abstract}

Keywords: Yoga; Spirituality; Intentions; Physical activity; Psychological wellbeing

\section{Introduction}

\section{The yoga boom in western society}

Over the past few decades, the Eastern movement form of yoga has become increasingly more prevalent in the Western world. Approximately 14.9 million Americans and half a million Britons are estimated to incorporate some form of this practice into their lives [1,2]. Given that yoga is now widely available, from being offered in yoga centres to shopping malls, this should come as no surprise. Perhaps what is more remarkable is the dissemination of this Eastern practice in legitimate socio-cultural Western environments, such as businesses, hospitals, and schools [3]. Moreover, the end of the $20^{\text {th }}$ century saw the advent of yoga as a commercialised multimillion dollar business, with postures, sequences, and styles being patented by companies and used to advertise a plethora of commodities, ranging from yoghurt to mobile phones [4]. In fact, recent data has suggested that American yoga practitioners expend up to 5.7 billion dollars per annum on yoga classes, products, and retreats [5]. Such observations led Campbell to postulate: Paradoxically, it is possible that just at the point when the rest of the world seems intent on imitating the Western way of life; the West itself is actually turning away from its own historic roots and embracing an Eastern outlook [6].

\section{Western yoga for physical fitness and therapy}

In particular, the Western manifestation of this Eastern practice appears to focus primarily on the physical forms of yoga. Endorsing this view, Singleton [4] argues that yoga in Western society is synonymous with the practice of asana (posture), and such asana-centred yoga sessions are easily accessible in almost every city in the West. Supporting this claim, in the majority of British contexts, yoga appears to be practised as part of a physical fitness regime, with the knowledge that asana improves flexibility and strength. For example, yoga was classified as a 'keep fit' lifestyle practice, alongside dance exercise and aerobics, in the UK's " 2002 General Household Survey" ; this category of leisure activities was rated as third most popular, and most regularly practised, after walking and swimming [7,8]. Further epitomising the physical nature of Western yoga practice is the accreditation of the British Wheel of Yoga (the UK governing body for yoga) by the UK Sports Council [2].

Whilst some practitioners consider asana to constitute an alternative form of exercise, others are drawn to this postural yoga as they believe it to offer an effective form of physical therapy to 'fix' illnesses, injuries, and remedial conditions. Nurturing these beliefs, promotional leaflets which are distributed by local clubs often describe yoga as a practice which "can be helpful to correct muscular skeletal imbalances, develop core strength and flexibility, improve breathing patterns, release stress and tension, increase energy and vitality..." [9]. In order to evaluate such body-mind transformational proposals, clinical trials have recently attempted to measure the effectiveness of yoga in treating a vast array of psychological and medical conditions, varying from reforming juvenile offenders to relieving back pain [10].

Although such research has been useful in elucidating the effectiveness of yoga as a physical fitness and therapeutic intervention technique, it has limited the definition of this Eastern form to a discrete sequence of postures and movements [10]. This allows for yoga practice to be interpreted as a means of physical therapy. However, Moliver et al. [11] argue that, for the most part, yoga was not traditionally practised for the purpose of curing defined pathologies but rather to boost lifelong spiritual and physical wellbeing.

*Corresponding author: Itai Ivtzan, Department of Psychology, University of East London, UK, Tel: +44 (0)208223 4384; E-mail: i.ivtzan@uel.ac.uk

Received August 06, 2015; Accepted August 07, 2015; Published August 13 2015

Citation: Ivtzan I, Jegatheeswaran S (2015) The Yoga Boom in Western Society: Practitioners' Spiritual vs. Physical Intentions and Their Impact on Psychological Wellbeing. J Yoga Phys Ther 5: 204. doi:10.4172/2157-7595.1000204

Copyright: ( 2015 Ivtzan I, et al. This is an open-access article distributed under the terms of the Creative Commons Attribution License, which permits unrestricted use, distribution, and reproduction in any medium, provided the original author and source are credited. 


\section{Spiritual differences between western and eastern yoga practices}

Yoga, as it originated over five thousand years ago on the Indian subcontinent, can be described as a contemplative discipline which integrates both mental and physical practices with the ultimate goal of attaining paramatman (pure consciousness) $[12,13]$. This ancient Indian practice was first conceived by rishis (sages) who were in search of a blissful state; this became known as "yoga", embodying the concept of unity between the body, mind, and spirit [14]. The rishis were able to experience yoga and attain samadhi (enlightenment) by altering their identification from the mind and body towards the Divine [15].

Yet, the Western literature offers almost no reference to the "yogic experience" acquired by commanding such modifications of the mind. Whilst this ancient Indian philosophy, that is yoga, is deeply rooted in spirituality-promoting components, including niyama (selfdiscipline), pratyahara (detachment from the senses), and dhyana (meditation), Western yoga is, in large, restricted to the practice of asana [14]. Yet, Singleton [4] asserts that there is, at best, limited evidence suggesting that asana was ever a fundamental component of any kind of traditional, Indian yoga practice. Instead, he contends that this rising asana-centred practice is predominantly shaped by a desire for European bodybuilding, and American and European women's gymnastics movements in the early twentieth century.

However, contrary to Singleton's [4] argument, Gannon \& Life [16] believes that Westerners, like the rishis, have the ability to practise yoga with the primary objective of attaining samadhi. Embracing this intense mystical journey towards enlightenment, they developed a novel "brand" of yoga in New York City in the mid-80s, called Jivamukti Yoga, which attempts to unite the spiritual, psychological, and physical components of traditional yoga for practitioners of the West. Gannon and Life [16] mirror the Indian system of yoga by translating the traditional Sanskrit teachings in a way which is compatible with "Western minds". Thus, through the sustained practices of chanting, intense meditation, and sacred readings, Jivamukti Yoga cultures growth with a continued dedication to yoga as a spiritual practice. This debate regarding the portrayal of yoga in Western society could be attributed to the fact that both the concepts of spirituality and health and fitness can be interpreted and defined in various ways. Bray [17] postulates that "if different conceptions of health coexist, then we need to ask who seeks or may seek which form" [10]. This idea that fitness and health are comparative social constructs implies that yoga should be situated within particular cultural and social frameworks which allow practitioners to offer their own definitions of what yoga signifies in their lives and what drives them to practice.

\section{A different perspective: individuals' intentions to practice}

Indeed, different practitioners comprehend yoga in different ways. Whilst some attend classes to "build stomach muscles", others practice yoga in the hope of progressing "more rapidly toward enlightenment" [10]. This highlights the extent to which individual experiences of yoga are flexible and fluid; whilst the former indicates a more physical intention to practice, the latter reflects a practice which is more spiritual in nature. The striking disparity between these two practitioners' motivations stresses the need to gather evidence beyond advertisements by local clubs and commercialized media representations in order to fully understand how and why yoga is practiced in Western cultures. Furthermore, whilst empirical studies have highlighted the therapeutic, psychophysiological, and occasionally even spiritual, benefits of yoga, they have not endeavored to elucidate the deeper meanings and motivations underlying Western practitioners' fascination for, and commitment to, this particular practice.

The present study aimed to conduct quantitative research exploring whether practitioners in the West begin and continue to practice yoga for spiritual and/or physical fitness purposes. This study welcomed practitioners devoted to any type of yoga practice. Furthermore, respondents with varied levels of experience were recruited, ranging from yoga teachers with relevant training qualifications to practitioners with less than one year of experience. The purpose was to create a diverse sample set which would be representative of the wider population of yoga practitioners spanning the Western world.

\section{Transformation of intentions: The role of time}

An important inference from Hasselle-Newcombe's [15] is that intentions, the practitioner's underlying motivations, are dynamic, with certain motivations becoming more salient to yoga practitioners as their practice becomes more established. Shapiro [18] reflected that as individuals progress in their practice, their intentions "shift along a continuum from self-regulation, to self-exploration, and finally to self-liberation". Self-regulation refers to one's control over cognitive processing (stress control for example), self-exploration describes one's investigation and knowledge of the self, and self-liberation can be described as reaching a transcendental state in which one experiences freedom from the sense of being a separate self. The idea is that as practice becomes more regular and grounded in the practitioner's life, they will shift from attaining motivations such as stress management to embarking on a spiritual journey towards enlightenment. Thus, the present study also investigated whether intentions to practice yoga become more spiritual over time, from when practitioners first begin their practice to their continued motivations to practice. In addition, this study investigated whether there are differences between practitioners' initial and continued physical intentions to practice yoga.

\section{Yoga practice and psychological wellbeing}

Whilst on one hand, we have seen that variables such as time appear to significantly impact practitioners' intentions and thus the outcome of their practice, on the other hand, there are variables which are influenced by yoga practice itself [19]. One such variable which has repeatedly presented in the literature is practitioners' psychological wellbeing. Here, wellbeing does not encompass the absence of difficulties in one's life, but rather the presence of positive qualities experienced throughout one's life, including happiness, meaning, vitality, and selfactualisation [20]. This positive psychological wellness is comprised of both eudemonic and hedonic entities, both of which have been strongly linked to the practice of yoga [21]. Whilst eudemonia is built on ideas of growth and meaning in life, hedonia reflects the idea of subjective wellbeing, which includes concepts of elevated positive affect and satisfaction with life.

A number of studies have indicated that yoga-based interventions are successful in enhancing various aspects of hedonic wellbeing in young adults [22]. More specifically, whilst some have revealed that yoga practice correlates with high levels of life satisfaction others have highlighted the effects of yoga in boosting vitality and enjoyment levels [23,24]. In terms of eudemonic wellbeing, research appears to support the notion that yoga practice can promote significant increases in meaning in life. For example, a study by Ivtzan and Papantoniou [25] reported that there is a significant positive association between how experienced yoga practitioners are and their reported meaning in life. Furthermore, Hasselle-Newcombe's [14] showed that $85 \%$ of 
the respondents ascribed an enhanced sense of meaning in their lives to their yoga practice. Many practitioners in this study also offered qualitative reflections on their personal experiences of eudemonic wellbeing, encompassing ideas such as yoga giving their life a rootedness and sense of purpose, and yoga forming part of their self-identity.

Yet, whilst these scholars have reported respondents' enhanced levels of wellbeing as a result of their yoga experience, there has been no systematic attempt made to explore how different intentions to practise yoga affect practitioners' psychological wellbeing. On one hand, the notion that meaning is a fundamental expression of spiritual development [26] suggests that, relative to practitioners who are driven by physical fitness aesthetics, individuals who practise yoga with spiritual intentions have a clearer sense of meaning and purpose in their lives. On the other hand, although the underlying mechanisms are yet to be elucidated [27] it has also been suggested that physical fitness and exercise promote psychological wellbeing [28]. Thus, whilst both spirituality and physical exercise appear to promote positive wellness, it appears ambiguous, at present, as to how those who practise yoga with physical fitness intentions and those who practise with spiritual intentions would vary in terms of the psychological benefits they experience.

However, this is a valuable avenue of research when considering how yoga is perceived and practised in Western society. If practitioners who are motivated by spiritual development have a greater level of psychological wellbeing relative to those who are driven to practise yoga as an alternative form of exercise, or vice versa, this would have strong implications for the way in which yoga should be practised in Western society; that is, whether the West should continue to practise yoga as a primarily postural practice, or whether a greater emphasis should be placed on the traditional, spiritual experiences of yoga, as exemplified by Jivamukti yoga $[4,15]$. In order to provide a greater insight into these implications, this study also examined whether there are marked differences in psychological wellbeing levels between Westerners who practise yoga with spiritual intentions and those who practise with physical fitness intentions.

\section{Method}

\section{Participants}

A total of 235 British yoga practitioners participated in this study. The sample was comprised of 192 female and 43 male participants, whose ages ranged from $18-70$ years $\left(\mathrm{M}_{\text {age }}=40.25\right.$ years, $\left.\mathrm{SD}=12.42\right)$; the minimum age requirement to participate was 18 years. The criteria to take part also involved regular yoga practice equating to at least once a week on average; of those who participated, $31 \%$ practised yoga every day, $35 \%$ at least three times a week on average, and $34 \%$ at least once a week on average. Participants also varied in terms of their level of yoga experience: $11 \%$ had been practising for less than one year, $55 \%$ for up to ten years, $23 \%$ for $10-20$ years, and $11 \%$ for more than 20 years; 11 practitioners of this sample set also reported that they were qualified yoga teachers.

\section{Apparatus and Measures}

Each participant completed a questionnaire, which was divided into three main parts:

Demographics: The first section was concerned with basic demographic information, such as participants' gender, age, and educational qualifications.
Yoga: The second section focused on respondents' experience of, and personal intentions for, practicing yoga:

Yoga experience: Practitioners provided details of the extent and frequency of their practice.

Yoga intentions: Respondents answered two major questions relating to their motivations for initially beginning and later adhering to their yoga practice. The first question read "how important were the following possible motivations for you when you FIRST began yoga?" and the second question read "when considering why you continue to practice yoga, how important to you is the following aspects?" Both of these questions were followed by a list of six Likert-type statements, of which three were strongly associated with spiritual intentions (e.g. "spiritual development") and three with physical intentions (e.g. "an alternative form of physical exercise"). These questions and the Likerttype statements were extracted from the original questionnaire used in Hasselle-Newcombe's [2]. Each intention item was scored on a 7-point scale, from one (definitely not important) to seven (definitely very important); respondents were asked to circle the point on each scale which they felt was most appropriate to them. For comparative purposes, the same six Likert-type statements were used for both questions.

\section{Psychological wellbeing:}

Satisfaction with life: Respondents' satisfaction with life was measured using the satisfaction with life scale (SWLS), which is comprised of five broad statements (e.g. "the conditions of my life are excellent"). Respondents had to indicate the extent to which they endorsed each of the five items by rating them using a 7-point scale, from disagreement to agreement (From $1=$ strongly disagree, to $7=$ strongly agree) [29]. Each respondent was given a total satisfaction with life score by adding together the ratings given for each of the 5 statements.

Subjective happiness: Practitioners' subjective happiness was measured using the subjective happiness scale (SHS) [30]. Items in this scale were scored on a scale between one-to-seven. For each statement, they were asked to select the point on the scale which they felt was most appropriate in describing their wellbeing. Each respondent was given a total subjective happiness score by combining their ratings for all items.

Meaning in life: Practitioners' meaning in life was measured as a reflection of their eudaimonic wellbeing, using the meaning in life questionnaire (MLQ) [31]. They were requested to respond to each item as accurately and truthfully as possible, bearing in mind that as all of the questions were subjective in nature there were no right or wrong answers. They scored the items using a 7-point scale indicating their level of agreement with each (From $1=$ absolutely untrue, to $7=$ absolutely true). The ratings for each item were added together to give each respondent an overall meaning in life score.

\section{Procedure}

Data for this study was collected via hard copies of the questionnaire and an online survey replicating the paper version. The questionnaire was distributed as a hard copy to yoga practitioners who attended the Om Yoga Show (making up 72\% of the sample set), which took place at the Olympia National from 25th - 27th October 2013. As the largest annual yoga event in Europe, this show offered the opportunity to gather participants with a diverse range of yoga experience and a 
Citation: Ivtzan I, Jegatheeswaran S (2015) The Yoga Boom in Western Society: Practitioners' Spiritual vs. Physical Intentions and Their Impact on Psychological Wellbeing. J Yoga Phys Ther 5: 204. doi:10.4172/2157-7595.1000204

Page 4 of 7

varied set of intentions and interests. The same questionnaire was also administered as an online questionnaire (making up the remaining $28 \%$ of the sample).

\section{Results}

\section{Intentions}

Statistical analyses were conducted to test the main hypotheses predicted in this study (Table 1) for the paired samples t-tests); an alpha level of 0.05 was used for all statistical tests. Firstly, as predicted, it was found that practitioners' physical intentions $(M=16.99$; $S D=3.53)$ were significantly higher than their spiritual intentions $(\mathrm{M}=11.87$; $\mathrm{SD}$ $=5.05)$ when considering why they first took up the practice of yoga, $\mathrm{t}(234)=12.15, \mathrm{p}<0.001$ (one-tailed). The effect size for this analysis ( $\mathrm{d}=0.79)$ is moderate-to-large, following Cohen's [15] convention for effect sizes. Moreover, confirming the extension of this hypothesis, relating to continued intentions, it was found that practitioners reported significantly higher physical intentions $(M=17.33 ; \mathrm{SD}=3.74)$ than spiritual intentions $(\mathrm{M}=16.11 ; \mathrm{SD}=4.29)$ when considering their current motivations for adhering to their yoga practice, $\mathrm{t}(234)=3.11$, $\mathrm{p}=0.001$ (one-tailed). Although this result was statistically significant, the effect size was smaller $(d=0.20)$.

Another statistically significant finding was that spiritual intentions increase over time, from when practitioners initially take up yoga $(M$ $=11.87 ; \mathrm{SD}=5.05)$ to when their practice becomes more established $(\mathrm{M}=16.11 ; \mathrm{SD}=4.29), \mathrm{t}(234)=-12.52, \mathrm{p}<0.001$ (one-tailed). This is in line with the hypothesis that spiritual motivations become more salient as a practitioner continues with their yoga practice. Furthermore, the effect size for this analysis $(\mathrm{d}=.82)$ was found to exceed Cohen's [15] classification for a large effect size $(\mathrm{d}=0.80)$. However, the t-test conducted to see if there was a difference between initial and continued physical intentions did not reach statistical significance, $\mathrm{t}(234)=-1.62$, $\mathrm{p}=0.107 ; \mathrm{d}=0.12$. This indicates that practitioners' physical intentions did not change over time from when they first took up the practice to their motivations for continuing.

\section{Psychological wellbeing}

In terms of psychological wellness, a significant difference was found between overall wellbeing levels between practitioners who were classified as having continued spiritual intentions and those who were categorised as having continued physical intentions, $\mathrm{F}(1,195)=$ 29.04, $p<0.001$. As shown in Figure 1, practitioners who reported more spiritual than physical intentions, when considering why they continue to practice yoga, had significantly higher levels of overall psychological wellbeing $(\mathrm{M}=91.39, \mathrm{SD}=12.42)$, compared to those who reported more physical than spiritual intentions $(\mathrm{M}=79.25, \mathrm{SD}=16.88)$.

Similar results are found when the overall wellbeing score is broken down into its components. As can be seen from the one-way ANOVA tests shown in Table 2, both eudaimonic and hedonic wellbeing also differed significantly between practitioners with physical intentions and those with spiritual intentions. On the MLQ, out of a maximum score of 70, respondents with spiritual intentions had an average rating of 51.89 ( $\mathrm{SD}=9.48$ ), whilst those with physical intentions only scored an average of $43.88(\mathrm{SD}=12.29)$. When ratings for the SWLS and SHS were combined to give an overall subjective wellbeing score, those with spiritual intentions had a mean score of $39.49(S D=5.33)$, whilst those with physical intentions only had an average score of $35.38(\mathrm{SD}=6.95)$. Table 2 also shows that the effect size was greatest when scores on all three scales were combined together $(\mathrm{d}=0.82$; large effect size $)$, and lowest when the SWLS + SHS scores were combined as a measure of

\begin{tabular}{|c|c|c|c|c|}
\hline Intentions & $\mathbf{N}$ & $\mathbf{t}$ & $p$ & d \\
\hline (1) Initial physical vs. Initial spiritual $_{\text {spita }}$ & 235 & 12.15 & $<0.001^{* *}$ & 0.79 \\
\hline (2) Continued $\mathrm{d}_{\text {physical }}$ vs. Continued $\mathrm{spiritual}$ & 235 & 3.11 & $0.001^{*}$ & 0.20 \\
\hline (3) Spiritual initial $_{\text {VS. Spiritual }}$ continued $_{\text {. }}$ & 235 & -12.52 & $<0.001^{* *}$ & 0.82 \\
\hline (4) Physical $_{\text {initial }}$ vs. Physical continued $_{\text {. }}$ & 235 & -1.62 & 0.107 & 0.12 \\
\hline
\end{tabular}

Note: $\mathrm{N}=$ number of participants; $\mathrm{d}=$ Cohen's $\mathrm{d}$ effect size, ${ }^{*} \mathrm{p}<0.01$, one-tailed, ${ }^{* *} p<0.001$, one-tailed

Table 1: Paired Samples t-tests looking at Initial and Continued, Spiritual and Physical Intentions.

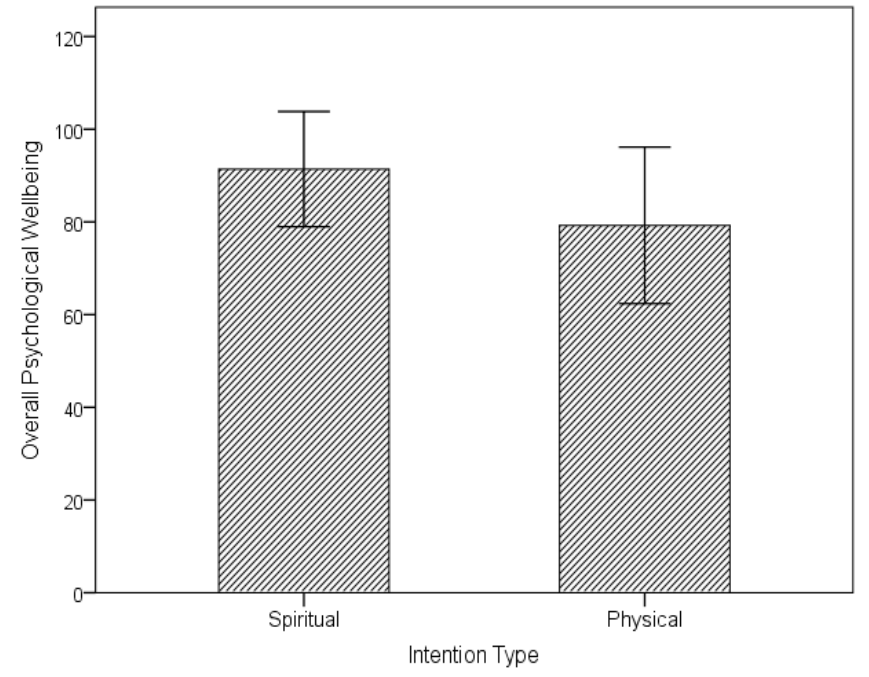

Figure 1: Overall psychological wellbeing as a function of continued intention type. Mean overall wellbeing scores (SWLS + SHS + MLQ) for practitioners classified as continued spiritual intentions and those grouped as having continued physical intentions. Error bars indicate standard deviation adjusted to eliminate between-subjects variance (+/- 1 standard deviation).

\begin{tabular}{|c|c|c|c|c|}
\hline Psychological Wellbeing & $\mathbf{d f}_{\mathbf{1}}, \mathbf{d f}_{\mathbf{2}}$ & $\mathbf{F}$ & $\mathbf{p}$ & $\mathbf{d}$ \\
\hline Overall & 1,195 & 29.04 & $<0.001^{* *}$ & 0.82 \\
\hline Subjective wellbeing & 1,195 & 19.30 & $<0.001^{* *}$ & 0.66 \\
\hline Meaning in life & 1,195 & 23.33 & $<0.001^{* *}$ & 0.73 \\
\hline
\end{tabular}

Notes: $\mathrm{df}=$ degrees of freedom; $\mathrm{F}=\mathrm{F}$-ratio; $\mathrm{d}=$ Cohen's $\mathrm{d}$ effect size. Overal wellbeing = SWLS + SHS + MLQ; subjective wellness = SWLS + SHS; meaning in life $=M L Q,{ }^{* *} p<0.001$

Table 2: ANOVA Table looking at how Psychological Wellbeing varies as a function of In:tention type.

hedonic wellbeing $(\mathrm{d}=0.66)$. Yet, according to Cohen's convention [15], this still constituted a moderate effect size. Overall, as predicted, these results show that there is significant evidence to suggest that practitioners' psychological wellbeing varies as a function of their current intention type; spiritual intensions yield significantly higher levels of wellbeing compared with physical intentions [32].

\section{Discussion}

\section{Overview}

This study predicted that yoga practitioners would report more salient physical intentions, relative to spiritual intentions, for both their initial and continued motivations to practice yoga. Furthermore, it was hypothesised that practitioners' intentions would become more spiritual over time, and that their physically-oriented intentions would 
vary significantly between initial and continued practice. Finally, in terms of psychological wellbeing, it was predicted that respondents with continued spiritual and physical intentions would cultivate significantly different levels of psychological wellbeing. The results of this study supported all but one hypothesis, that is, the prediction that there would be a significant difference between practitioners' physical intentions from initial to continued practice.

\section{Evidence for physical intentions to practice yoga}

Firstly, this study revealed that individuals are more driven to practice yoga for its physical virtues than for the purpose of acquiring spiritual development. This result supports the work of HasselleNewcombe [2], who found that the majority of respondents in her study reported that their initial motivations for taking up the practice involved physical concepts such as perceiving yoga to be a type of exercise routine. She also reported that individuals had high levels of physically-oriented intentions when considering why they continue to practice yoga; particular ideas of maintaining flexibility and fitness seemed to be key incentives. Similarly, this study found themes of stretching and strength building to be highly imperative to individuals' investment in yoga.

Furthermore, the current study found that such physical fitness intentions are more salient to practitioners than spiritual intentions both when they begin their practice and when reasoning their motivations for continuing with their practice. This provides support for qualitative research exploring Western practitioners' physical intentions to take to the mat. For example, Lewis [10] reported that practitioners repeatedly refer to concepts of movement and challenge, implicating yoga as a form of workout. This notion is mirrored in the results of this study, which showed that practitioners are highly driven to practise yoga for the purposes of developing physical strength and engaging in regular exercise.

However, this result offers no account of the spiritual yearning expressed by individuals in Brown and Leledaki's [9] study. Interviewees in their study reported Orientalist perceptions and a burning desire towards the exotic other, from a young age, which implies that individuals should report more spiritual intentions even from the point of their initial practice. However, for a highly significant proportion of the respondents who took part in the present study, this was not the case. Therefore, whilst Brown and Leledaki [9] attempted to explain the manifestation of eastern movement forms in the West, their methodological approach of conducting in-depth life history interviews on a small set of long-term practitioners may reductively reflect the particular intentions of a limited sample which is unrepresentative of the general population of practitioners.

\section{Transformation of intentions from initial to continued practice}

Although yoga is undoubtedly practised as an enhanced physical fitness activity amongst many of its practitioners in the West, this study has provided some pertinent findings regarding the way in which yoga practice can transform over time. Firstly, it was found that practitioners' spiritual intentions become more salient from when they first begin practice to when their practice becomes more established, indicating that after individuals take up the practice of yoga they embark on a journey of spiritual development. This supports Shapiro's [18] work on intentions, which suggests that as individuals invest more time and effort into regular practice, their motivations for doing so will shift from a focus on physiological and emotional regulation to a desire to achieve Samadhi [18]. Thus, Shapiro's [18] theory of intentions shifting along a continuum can be extended beyond the specific practice of meditation to the wider practice of yoga. This is the blossoming of spiritual intentions over time. One of the study's limitations is the fact that it was not measured how long it took practitioners to shift from one intention to another. This could be an interesting avenue for future research.

Whilst practitioners' spiritual intentions evolved over time, the current study revealed that there was no significant difference between their physical intentions from when they first began yoga to their current practice of it. Although physical intentions do not decrease, they remain constant; this also strengthens our understanding of Shapiro's [18] work, suggesting that practitioners become more focused on their spiritual journey as their practice progresses, abandoning any growth of interest in their physical intentions.

\section{Theoretical insight into the debate: How yoga is practiced in the west}

As suggested by Kabat-Zinn [33], intentions provide a benchmark for one's potential, and tend to correlate with the outcomes of practice. Thus, the implications of this intention research are widespread in the domain of yoga literature, specifically regarding the debate of how yoga is practiced in the Western world $[33,18]$.

The result that individuals' intentions to practice yoga are more physical in nature both when they begin and continue with their practice suggests that Singleton [4] was correct in stating that yoga practice in the West is synonymous with the practice of asana. According to this study, the rise of asana practice appears to be accounted for by practitioners' enthusiasm towards the notion that postures and poses result in greater levels of stretching, flexibility, and physical strength. The implication is that practitioners are genuinely more driven by the aesthetic benefits of yoga, such as developing a slim waistline and building stomach muscles, as a practitioner in Lewis' [10] study reported. Such ideas suggest that there are indeed striking disparities between the conceptualisation of yoga practice in the West and the historically Indian tradition of yoga, as conceived and practised by rishis over 5,000 years ago.

However, revelations regarding the transformation of intentions over time offer a somewhat divergent approach to this debate. Whilst practitioners are undeniably enticed by the physical attributes of yoga, once their practice becomes more grounded their spiritual involvement strengthens. This shows that Western yoga, like classical Indian yoga, cultivates the opportunity for spiritual growth. In suggesting this, the present study also provides credibility to studies conducted by Büssing et al. [34] and Wilson and Spencer [35] who demonstrated that Western yoga can promote transformational processes of spirituality and transcendence amongst its practitioners. Furthermore, the fact that physical regulation does not become a more prominent intention with time suggests that even in Western yoga practice it is the spiritual experience that is growing and evolving, and not the physical one.

Thus, whilst Singleton [4] may have been correct in predicting practitioners' initial motivations for taking to the mat, his claim that Western yoga is primarily asana-centred does not account for the increased salience of spirituality as practice becomes more established. Instead, this result is better fitted with the opposing argument of this debate, in which authors such as Gannon and Life [16] have shown that Western yoga practice, like the original Eastern yoga tradition, can cultivate states of spirituality. This suggests that Singleton's [4] account 
of asana practice in Western culture is, to some extent, a reductionist one that does not capture the full meaning of the practice which is experienced by its practitioners. Moreover, the significant role of time in transforming practitioners' intentions shows that the debate is more complex than initially posited.

Finally, this debate should take into account the question of different types of yoga. As discussed in the introduction, certain types of yoga are more aligned with the original spiritual intention of the yogic practice. The contemporary Jivamuktiy yoga is one example while Kundalini yoga is another. Other types of yoga might be more focused on the physical aspect of the practice; Bikram yoga as an example. Might it be that certain types of yoga encourage certain changes in the practitioner while other types lead to a different kind of transformation? Or perhaps the practitioner's intention lead to the choice of particular yoga types? These questions are outside the scope of this paper and yet we found them important to raise for future research in the area.

\section{Yoga practice and psychological wellbeing}

Another important avenue of research explored in this study concerned the psychological wellbeing of yoga practitioners. It was found that those who practise yoga with spirituality in mind had greater levels of positive wellness relative to those who practised for the purpose of gaining physical fitness and flexibility. When psychological wellness was divided into hedonic and eudaimonic wellbeing, this effect still persisted. In illustrating these results, the present research supports several existing studies.

Firstly, the result that yoga practice can cultivate enhanced levels of satisfaction in life and subjective happiness supports studies indicating that yoga-based interventions can promote hedonic wellbeing. More specifically, whilst respondents who scored high on the SWLS lend support to Brazier et al.'s [23] research findings showing a strong correlation between yoga practice and high levels of satisfaction with life, those who expressed high ratings on the SHS offer consistency with the results of Ghoncheh and Smith's [24] study, in which practitioners reported boosted energy and enjoyment levels as a result of their practice.

Moreover, this study has demonstrated that regular engagement with yoga practice can increase individuals' eudaimonic wellbeing. This is in line with reports from Hasselle-Newcombe's [2] study, in which the vast majority of respondents claimed that their yogic experiences enhanced their sense of meaning in life. Furthermore, this result supports Ivtzan and Papantoniou's [25] finding that practitioners can develop higher levels of meaning in life as a result of their yoga practice. The critical finding that individuals with spiritual intentions experienced greater eudaimonic wellbeing than those with physical intentions is also consistent with their explanations. They posited that spiritual aspects of yoga urge individuals to seek deeper explorations of meaning in their life, resulting in a more purposeful and goal-oriented existence. This spiritual route of enhanced personal growth provides a plausible explanation for why those who endorse spiritual intentions reported significantly higher levels of psychological wellbeing, particularly in terms of meaning in life, than those with physical fitness motivations.

This thread between spirituality and meaning may also explain why the wellbeing effect size between the two intention groups was larger for eudaimonic wellbeing relative to hedonic wellbeing. Whilst both types of psychological wellbeing varied significantly as a function of practitioners' intention type, there is reason to believe that spiritual intentions to practise yoga cultivate higher levels of meaning in life than subjective wellbeing. This is because spirituality drives individuals to set themselves goals and search for meaning, via practices of selfawareness, self-discipline, and self-control [25].

\section{Practical implications of the debate: The importance of psychological wellbeing}

The finding that individuals who practise yoga with spiritual intentions have higher levels of self-reported psychological wellbeing compared to individuals who practise yoga with physical intentions has important practical implications regarding the way in which yoga should be conceptualised in the future. It may be the case that yoga institutions and governing bodies in the West should place a greater emphasis on the Indian-rooted spiritual aspect of yoga in an attempt to promote its psychological benefits amongst practitioners. Such practices which actively attempt to parallel the Indian spiritual system of yoga should become more widespread in order to maximise positive wellness amongst Westerners who invest in the practice of yoga. Furthermore, the everyday psychological gains of spiritual yoga practice should be made more explicit through media representations and promotional advertisements in order for lay perceptions of yoga to shift from a primarily fitness and health promoting intervention to a practice which enriches one's spiritual and personal growth.

\section{Directions for future research}

In order to eliminate the possibility of implicit memory distortions in individuals' retrospective ratings of their initial intentions, a longitudinal design might be more desirable in future research. This would require practitioners to rate their intentions for taking to the mat at the start of their practice and then again once their practice has become more established. Such prospective designs would also allow for the researcher to manipulate the time period between practitioners' 'initial' and 'continued' practice. Furthermore, in order to gain an even deeper understanding of how intentions transform over time, respondents could fill out the questionnaire at several points during their practice, with equal intervals between each (e.g. every three months), in order to examine at what point in time a practitioner's intentions shift from a focus on self-regulation to self-exploration, and then from self-exploration to self-liberation [17]

One specific avenue of future research in which a longitudinal design could be used, involves an exploration of health-related intentions to practice yoga. Although this study chose to focus on the physical fitness intentions of practitioners, another dimension of Western yoga practice which appears to deviate from the typically spiritual Indian practice involves health-related motivations. Whilst numerous studies have investigated the physiological benefits of practicing yoga, authors such as Moliver et al. [11] have argued that yoga was never traditionally practiced for the purpose of curing specific ailments. Thus, research could be conducted to examine practitioners' perceptions of yoga as a health-improving intervention, and how individuals with such healthrelated incentives differ in terms of psychological wellbeing compared to those with spiritual intentions.

Finally, cross-cultural research would produce pertinent findings in elucidating this debate. In particular, research testing whether Indian yoga practitioners have significantly higher levels of spiritual intentions than Western practitioners would be highly beneficial. This would offer an opportunity to explore whether the difference between Indian yoga and Western yoga is as stark as some scholars imply, or whether the culture of yoga is changing significantly across the world, including in its birthplace of India. 
Citation: Ivtzan I, Jegatheeswaran S (2015) The Yoga Boom in Western Society: Practitioners' Spiritual vs. Physical Intentions and Their Impact on Psychological Wellbeing. J Yoga Phys Ther 5: 204. doi:10.4172/2157-7595.1000204

Page 7 of 7

\section{Conclusion}

Paradoxically, whilst the rest of the world is striving to parallel the fast-paced lifestyle of the West, Western culture appears keen to adopt more tranquil Eastern practices such as yoga [6]. This yoga boom has served as the focal point of a controversial debate regarding the manifestation of yoga in the West, when compared against the classical Indian system. By focusing on Western practitioners' spiritual and physical intentions to commit to yoga, this study offered a unique perspective on the issue. Whilst Singleton's [4] account of asana was paralleled when practitioners considered their initial intentions to take to the mat, the gradual evolvement of their spiritual intentions, nurtured via sustained practice, supports Gannon and Life's [15] belief that Western yoga can also cultivate states of transcendence. More importantly, this study emphasises time as an essential dimension which was overlooked on both sides of the original debate. An additional avenue of research revealed that both eudaimonic and hedonic aspects of wellbeing are promoted amongst those who ascribe spiritual meanings and motivations to their practice. Thus, the present research concludes by accentuating the positive implications of practicing yoga with a spiritual bearing, synonymous to the historically Indian yogic tradition.

\section{References}

1. Hoyez A (2007) The 'world of yoga': The production and reproduction of therapeutic landscapes. Social Science \& Medicine 2: 1-19.

2. Newcombe SH (2005) Spirituality and 'mystical religion' in contemporary society: A case study of British practitioners of the lyengar method of yoga. Journal of Contemporary Religion 20: 305-321.

3. Bloom A (2006) Kick start KS2 success Times Educational Supplement.

4. Singleton M (2010) Yoga body: The origins of modern posture practice: Oxford University Press, New York.

5. Yogitha B, Ebnezar J (2014) Can Yoga be an Effective Tool in Managing Psychological Stress? Journal of Ethnomedicine 1: 1-7.

6. Campbell C (2007) The Easternization of the West: A thematic account of cultural change in the modern era. London, England: Paradigm.

7. Rickards L, Fox K, Roberts C, Fletcher L, Goddard E,at al. (2004) Living in Britain: Results from the 2002 general household survey.

8. Sport England (2002). Women in sport factsheet.

9. Brown D, Leledaki A (2010) Eastern movement forms as body-self transforming cultural practices in the West: Towards a sociological perspective. Cultural Sociology 4: 123-154.

10. Lewis CS (2008) Life chances and wellness: Meaning and motivation in the 'yoga market'. Sport in Society: Cultures, Commerce, Media, Politics 11: 535545.

11. Moliver N, Mika E M, Chartrand MS, Haussmann RE, Khalsa SBS, et al.(2013) Yoga experience as a predictor of psychological wellness in women over 45 years. International Journal of Yoga 6: 11-19.

12. De Michelis E (2005) A history of modern yoga: Patanjali and western esotericism. New York, NY: Continuum.

13. Nagendra HR (2008) Defining yoga. International Journal of Yoga 1: 43,44.

14. Turner EM, Turner C (2010) Does yoga shape body, mind and spiritual health and happiness: Differences between yoga practitioners and college students. International Journal of Yoga 3: 48-54.

15. Gannon S, Life D (2002) Jivamukti Yoga: Practices for liberating body and soul. New York, Ballantine Books.

16. Gannon S, Life D (2013) Jivamukti Yoga

17. Bray F (1999) Response to Alter's heaps of health. Current Anthropology Special Issue: Culture. A Second Chance? 40: 43-66.

18. Shapiro DH (1992) A preliminary study of long term meditators: Goals, effects, religious orientation, cognitions. Journal of Transpersonal Psychology 24: 23-39.

19. Shapiro S L, Carlson LE, Astin J A, Freedman B (2006) Mechanisms of mindfulness. Journal of Clinical Psychology 62: 373-386.

20. Lomas T, Hefferon K, Ivtzan I (2014) Applied Positive Psychology: Integrated Positive Practice. Sage Publications, London.

21. Straume LV, Vitters $\varnothing \mathrm{J}$ (2012) Happiness, inspiration and the fully functioning person: Separating hedonic and eudaimonic well-being in the workplace. Journal of Positive Psychology 7: 387-398.

22. Jadhav SG, Havalappanavar NB (2009) Effects of yoga interventions on anxiety and subjective well-being. Journal of the Indian Academy of Applied Psychology 35: 27-31.

23. Brazier A, Mulkins A, Verhoef $M(2006)$ Evaluating a yogic breathing and meditation intervention for individuals living with HIVIAIDS. American Journal of Health Promotion 20: 192-195.

24. Ghoncheh S, Smith JC (2004) Progressive muscle relaxation, yoga stretching, and $A B C$ relaxation theory. Journal of Clinical Psychology 60: 131-136.

25. Ivtzan I, Papantoniou A (2014) Yoga meets positive psychology: Examining the integration of hedonic (gratitude) and eudaimonic (meaning) wellbeing in relation to the extent of yoga practice. Journal of Bodywork \& Movement Therapies 18: 183-189.

26. Wong PTP (2012) The human quest for meaning: Theories, research, and applications, Routledge, New York.

27. Scully D, Kremer J, Meade MM, Graham R, Dudgeon K, et al. (1998) Physical exercise and psychological well-being: A critical review. British Journal of Sports Medicine 32: 111-120.

28. Penedo FJ, Dahn JR (2005) Exercise and well-being: A review of mental and physical health benefits associated with physical activity. Current Opinion in Psychiatry 18: 189-193.

29. Diener E, Emmons RA, Larsen RJ, Griffin S (1985) The satisfaction with life scale. Journal of Personality Assessment 49: 71-75.

30. Lyubomirsky S, Lepper H (1999) A measure of subjective happiness: Preliminary reliability and construct validation. Social Indicators Research 46: 137-155

31. Steger MF, Frazier P, Oishi S, Kaler M (2006) The meaning in life questionnaire: Assessing the presence of and search for meaning in life. Journal of Counseling Psychology 53: 80-93.

32. Cohen J (1988) Statistical power analysis for the behavioral sciences. Hillsdale $\mathrm{NJ}$ : Lawrence Earlbaum Associates.

33. Zinn JK (1990) Full catastrophe living: Using the wisdom of your body and mind to face stress, pain and illness. Delacorte, New York.

34. Büssing A, Hedtstück A, Khalsa SBS, Ostermann T, Heusser $P$, et al. (2012) Development of specific aspects of spirituality during a 6-month intensive yoga practice. Evidence-Based Complementary and Alternative Medicine: 981523.

35. Wilson SR, Spencer RC (1990) Intense personal experiences: Subjective effects, interpretations, and after-effects. Journal of Clinical Psychology 46 565-573.

Citation: Ivtzan I, Jegatheeswaran S (2015) The Yoga Boom in Western Society: Practitioners' Spiritual vs. Physical Intentions and Their Impact on Psychological Wellbeing. J Yoga Phys Ther 5: 204. doi:10.4172/21577595.1000204 